\title{
L'Europe des populismes : confluences et diversité
}

A Europe of populisms : convergence and diversity

Gilles Ivaldi et Andrej Zaslove

\section{(2) OpenEdition}

Journals

Édition électronique

URL : http://journals.openedition.org/ress/2996

DOI : 10.4000/ress.2996

ISSN : 1663-4446

Éditeur

Librairie Droz

Édition imprimée

Date de publication : 15 mai 2015

Pagination : 121-155

ISSN : 0048-8046

Référence électronique

Gilles Ivaldi et Andrej Zaslove, "L'Europe des populismes : confluences et diversité », Revue européenne des sciences sociales [En ligne], 53-1 | 2015, mis en ligne le 01 janvier 2019, consulté le 10 décembre 2020. URL : http://journals.openedition.org/ress/2996 ; DOI : https://doi.org/10.4000/ress.2996 


\title{
L'EUROPE DES POPULISMES: CONFLUENCES ET DIVERSITÉ
}

\author{
GILLES IVALDI \& ANDREJ ZASLOVE \\ Université de Nice Sophia Antipolis, URMIS - CNRS / \\ Radboud University (Nijmegen, Pays-Bas) - Institute for Management Research \\ gilles.ivaldi@unice.fr / a.zaslove@fm.ru.nl
}

\begin{abstract}
Résumé. Les élections européennes de mai 2014 ont été marquées par la poussée des partis populistes. À partir des données comparatives issues de l'enquête "Comparative Electoral Dynamics in the European Union in 2014", cet article examine les motivations du vote en faveur des partis populistes de gauche et de droite, et de leur rapport avec le projet d'intégration européenne dans les États membres contributeurs et bénéficiaires de l'UE. Nous trouvons des niveaux plus élevés de défiance politico-institutionnelle chez les supporters des partis populistes de gauche et de droite dans les deux groupes de pays, avec cependant des attitudes économiques et culturelles divergentes. Les supporters des populismes de gauche dans les pays bénéficiaires se révèlent plus eurosceptiques que leurs homologues des pays contributeurs. Dans les pays contributeurs, l'euro- scepticisme a un effet marginal sur le populisme de gauche mais il joue un rôle central dans la mobilisation par les partis populistes de droite, en lien avec le conservatisme culturel, l'opposition à la globalisation et le protectionnisme économique.
\end{abstract}

Mots-clés: droite radicale, élections européennes 2014, euroscepticisme, gauche radicale, populisme.

\begin{abstract}
The European Elections of 2014 were characterized by the rise of populist parties. Using cross-national data from the "Comparative Electoral Dynamics in the European Union in 2014" survey, this paper looks at what motivates voters to vote for both left and right-wing populists, and the relation between populist voting and EU integration across contributor and recipient EU member states. We find that supporters of populist parties on the left and right demonstrate higher levels of mistrust towards political institutions in both groups of countries, while diverging in terms of their economic and sociocultural attitudes. Left populist supporters in receiving countries tend to be more skeptical regarding EU integration than the populist left in contributing countries. In the latter, euroscepticism has a marginal effect on support for left wing populist parties while it shows a strong impact on right wing populist voting, in relation to cultural conservatism, anti-globalization and economic protectionism.
\end{abstract}

Keywords: European elections 2014, euroscepticism, populism, radical left, radical right. 
Les élections européennes de mai 20I4 ont été marquées par le déclin des grands partis de gouvernement et la poussée des forces périphériques. Dans des pays comme la France, le Royaume-Uni, le Danemark ou l'Autriche, ce phénomène s'est traduit pour l'essentiel par le soutien aux protagonistes établis de la droite radicale populiste, tels le Front national (FN), le Parti pour l'indépendance du Royaume-Uni (UKIP), le Parti du Peuple danois (DF) ou le Parti autrichien de la Liberté (FPÖ) (Ivaldi, 20I4). En Europe méridionale, nous avons assisté au succès de formations de gauche populiste, à l'image de SYRIZA (Coalition de la gauche radicale) en Grèce ou de Podemos en Espagne. De nouveaux acteurs ont émergé, enfin, à la marge du système politique européen, à l'instar d'Alternative für Deuschland (AfD) outre-Rhin ou du populisme post-matérialiste incarné par le Movimento Cinque Stelle ( $\mathrm{M}_{5} \mathrm{~S}$ ) de Beppe Grillo en Italie, dont la nature demeure pour l'heure sujette à débats.

L'essor de formations populistes à l'extrémité gauche de l'axe politique, parallèlement à la consolidation des partis de droite radicale, contribue à un renouveau de l'intérêt porté au phénomène populiste', dans un champ scientifique fortement balisé depuis le milieu des années 1980 par l’analyse du développement de la droite radicale (Mudde, 2007). À la lumière des élections européennes de 20I4, cet article propose de (re)poser la question des motivations du vote en faveur des partis populistes, plus spécifiquement des confluences mais également des divergences observables dans les dynamiques de soutien à ce type de formations dans l'espace européen. Nous nous intéressons en particulier aux facteurs qui unifient les populismes de gauche et de droite - et ceux qui les distinguent -, s'agissant notamment du rapport que ces mouvements entretiennent avec le projet d'intégration européenne. La littérature présente les partis de la gauche et de la droite radicales comme des forces d'opposition à l'Europe (Halikiopoulou et al., 20I2 ; Vasilopoulou, 20II). Toutefois, l'examen de ces partis et des motivations de leurs électorats permet d'envisager l'hétérogénéité de cet antagonisme vis-à-vis de l'Union européenne (UE) - essentiellement économique à gauche, par exemple, tandis que l'euroscepticisme des droites 
populistes s'articulerait plus spécifiquement sur des enjeux culturels. La prise en considération de cette diversité est essentielle à la compréhension de la signification de l'essor du populisme pour le processus d'intégration européenne.

Dans une première partie, nous proposons une brève revue de la littérature sur le populisme, qui nous fournit l'opportunité de formuler un ensemble d'hypothèses $\left(\mathrm{H}_{\mathrm{I}}-\mathrm{H} 9\right)$. Notre approche diffère cependant de la plupart des travaux existants en proposant une analyse conjointe des populismes de gauche et de droite dans une perspective comparative. À ce jour, la plupart des études relèvent uniquement de monographies ou bien se sont restreintes à l'analyse du populisme de droite en Europe ou à l'examen du populisme de gauche dans le contexte latino-américain. En outre, beaucoup de ces travaux se sont concentrés sur la question théorique de la définition du phénomène populiste. Cet article propose une approche empirique basée les données de l'enquête «Comparative Electoral Dynamics in the European Union in 20I4» (Sauger, Dehousse, Gouhou, 2015), qui nous permet d'examiner les facteurs de demande (demand-side) des populismes européens. Les implications de nos résultats pour la compréhension générale du phénomène populiste, de sa structuration dans le contexte de crise économique et de son impact sur l'avenir de l’UE sont discutées dans la dernière partie.

\section{EXPLIQUER LE(S) POPULISME(S)}

Les travaux sur le populisme peuvent être divisés en quatre groupes (Akkerman et Zaslove, 2014) : ceux qui voient dans le populisme un phénomène trop hétérogène pour être catégorisé (Ionescu et Gellner, 1969), ceux qui définissent le populisme essentiellement comme un style (Canovan, I999; Jagers et Walgrave, 2007; Moffitt et Tormey, 20I4), ceux qui le considèrent comme une stratégie (Weyland, 200I) et ceux, enfin, qui le définissent comme une idéologie peu substantielle ou «fine» (thin-centered ideology) (Mudde, 2004). Sans tenter de clore ici le débat, cette dernière perspective nous paraît présenter plusieurs avantages distincts (Akkerman et al., 20I4). Celui, tout d'abord, d'opérer à un niveau suffisant d'abstraction, qui rend possible l'importation du concept de populisme dans divers contextes nationaux ou régionaux - à l'image, notamment, du regard croisé sur le populisme en Amérique latine et 
en Europe -, et permet d'identifier les conditions nécessaires et suffisantes de sa définition (Mudde et Rovira Kaltwasser, 20I3). En second lieu, en postulant que le populisme doit prendre appui sur d'autres formes idéologiques, l'approche en termes d'idéologie fine permet d'identifier et d'éclairer une variété large de modalités de populisme: ainsi de ses variantes libérale, de droite radicale et socialiste. Enfin, en définissant le populisme comme un ensemble d'idées, ce cadre fournit les outils nécessaires à la mesure de sa présence ou de son absence dans l'offre programmatique des partis, dans leur communication politique et dans les attitudes de leurs électeurs (Akkerman et al., 20I4).

\section{DÉFINIR LE POPULISME}

La définition du populisme comme idéologie fine repose sur quatre caractéristiques: le populisme y est décrit comme la référence au peuple (Rooduijn et al., 20I4); il oppose un peuple «pur» à des «élites corrompues » (Mudde, 2004); cette opposition est manichéenne, en ce sens qu'elle décrit essentiellement une lutte entre le bien et le mal (Mudde, 2004; Hawkins, 2009) ; enfin, le focus est mis sur l'expression de la volonté populaire. Mudde résume ainsi la notion de populisme :

une idéologie qui considère la société comme étant au final séparée entre deux groupes homogènes et antagonistes, le peuple «pur» contre «l'élite corrompue», et qui considère que la politique devrait être l'expression de la volonté générale du peuple (2004, p. 543).

Si ces éléments composent l'ossature du populisme, ce dernier doit s'arrimer à d'autres idéologies. Dans le contexte européen contemporain, le débat se concentre pour l'essentiel, nous l'avons évoqué, sur trois différentes formes de populisme: celui de la droite radicale, sa variante socialiste/social-démocratique et enfin le populisme libéral. Au regard de l’objet du présent article, nous limitons notre revue de littérature aux deux premiers types. 


\section{GAUCHE ET DROITE POPULISTES}

Lapproche du populisme en termes d'idéologie fine permet la mesure du phénomène tant du point de vue de l'offre que de la demande, à travers l'étude des manifestes et des discours partisans ou, pour ce qui concerne plus directement notre propos, des attitudes des électeurs. Avec l'objectif d'identifier et de classifier les diverses formations populistes qui ont émergé en Europe, la littérature fournit un ensemble d'hypothèses quant à la structuration de la demande électorale pour ce type de partis.

Dans les termes du modèle proposé par Mudde (2004), le populisme devient précisément la variable unificatrice des acteurs de gauche et de droite, autour du centrage sur le peuple, de l'évaluation positive de ce dernier et de la perception négative de l'élite «corrompue » (Akkerman et al., 20I4; Akkerman et Zaslove, 20I4). À gauche de l'axe politique, la reconfiguration de la notion de peuple vise à établir une «chaine d'équivalence» au sein d'un ensemble d'acteurs, d'identités, de demandes et d'intérêts, en soulignant leur opposition à un «autre» commun, représenté par l'establishment politique et/ou l'élite néolibérale, divisant en définitive l'espace social en deux camps antagonistes (Stavrakakis et Katsambekis, 20I4, p. I29).

Empiriquement, le populisme peut être appréhendé à partir du rapport qu'entretiennent les électeurs avec les institutions et les élites politiques. Le degré d'insatisfaction politico-institutionnelle représente sans doute un prédicteur admissible du vote populiste, mais il demeure cependant une mesure par proxy. Gardant cette limitation à l'esprit, nous anticipons un effet positif de l'expression d'un mécontentement à l'encontre des élites et des institutions sur la propension à soutenir des partis de ce type, indépendamment de leur position sur l'axe gauche-droite (Akkerman et Zaslove, 2014)2.

2 Dans le cadre spécifique du scrutin européen, le modèle d'élection de «second ordre» popularisé par Reif et Schmitt (1980) met par ailleurs l'accent sur la tendance des électeurs à sanctionner les exécutifs en place et apporter leur soutien aux partis protestataires à la périphérie du système politique, en particulier les formations populistes. 
H. Les électeurs qui soutiennent les partis populistes de gauche et de droite présentent des niveaux plus élevés de défiance politique et institutionnelle que les électeurs des partis mainstream.

Si le paramètre commun unificateur demeure la référence à la notion de peuple, les idéologies sur lesquelles le populisme vient prendre appui agissent, à l'inverse, en tant qu'éléments de différentiation. Populismes de gauche et de droite divergent a priori sur la saillance qu'ils accordent aux enjeux socio-économiques et culturels et sur les positions qu'ils prennent à l'égard de ces derniers (Mudde et Rovira Kaltwasser, 20I3 ; Akkerman et Zaslove, 20I4). Ces dissimilitudes entre une forme «économiquement inclusive» et une variante «culturellement exclusive» sont au cœur de la distinction entre populisme de gauche et de droite qu'établissent, par exemple, Mudde et Rovira Kaltwasser (20I3). Ces auteurs fondent leurs conclusions sur l'examen croisé du populisme de droite en Europe et du populisme de gauche en Amérique latine, mais une distinction similaire peut, selon toute vraisemblance, être opérée pour l'Europe occidentale (De Lange et Rooduijn, 20I I ; Akkerman et Zaslove, 20I4). Dans le cadre de cet article, l'opérationnalisation de cette distinction invite à la formulation d'un certain nombre d'hypothèses plus spécifiquement relatives aux facteurs de demande électorale.

\section{I.LA DROITE RADICALE POPULISTE}

L’abondante littérature consacrée à la droite radicale populiste a mis en exergue la centralité du nativisme dans l'offre politique de ces partis (Mudde, 2007). Les formations de la droite radicale populiste s'opposent au multiculturalisme et, de manière croissante, se sont appropriées un agenda anti-Islam (Betz et Johnson, 2004). Les analyses des attitudes des électeurs de ces mouvements confirment très largement ces faits. L'opposition à l'immigration, nourrie de la perception d'une menace culturelle et de la crainte d'une compétition économique immigrée, demeure sans l'ombre d'un doute l'enjeu déterminant du vote de droite radicale populiste (Ivarsflaten, 2008 ; Oesch, 2008 ; Lucassen et Lubbers, 20I2; Akkerman et Zaslove, 20I4). 
H2. les électeurs des partis de la droite radicale populiste ont un plus fort degré de rejet de l'immigration comparés aux électeurs mainstream et aux électeurs de la gauche populiste.

Outre le nativisme, l'autoritarisme constitue un second enjeu d'offre partisane important pour ces partis, quand bien même cet aspect a été relativement plus négligé par la recherche comparative. Mudde (2007, p. 23) note à ce propos que les mouvements de la droite radicale populiste mettent l'accent sur le conservatisme moral et la nécessité de politiques répressives strictes dans la lutte contre la criminalité. Si ces deux formes attitudinales sont consubstantielles de la notion d'autoritarisme telle que définie par Adorno et al. (1950), les positions effectives des partis de la mouvance radicale populiste varient cependant dans l'espace et dans le temps (Akkerman et Zaslove, 20I4). Spierings et Zaslove (2015) montrent notamment une diversité de positions sur les questions familiales au sein de ces formations. En termes de demande, le degré d'autoritarisme varie également dans les électorats des droites radicales populistes et son effet demeure généralement moins ample que celui du nativisme. Nonobstant ce pouvoir explicatif plus limité, il est possible de postuler un niveau significatif d'attitudes autoritaires chez les supporters de ces partis, en anticipant des variations (Dunn, 20I3; Akkerman et Zaslove, 20I4).

H3. comparés aux électeurs mainstream et à ceux de la gauche populiste, les électeurs des droites radicales populistes sont plus conservateurs en matière sociétale et sont favorables à des politiques plus sévères dans le domaine de la lutte contre la criminalité. Ces niveaux varient selon les partis.

S’agissant de la dimension économique, en revanche, la littérature témoigne d'une plus grande indétermination quant à l'accent mis sur ce type d'enjeux et leur cadrage par les formations radicales populistes (Akkerman et Zaslove, 20I4). En termes d'offre programmatique, Rovny (20I3) souligne la faible saillance et le «brouillage» (blurring) des positions économiques de ces partis, qui tranchent avec l'importance et la lisibilité de leur programme sur les enjeux culturels. Des débats existent, par ailleurs, quant à la nature du corpus économique de la droite radicale populiste et la présence (ou non) de marqueurs libéraux (Kitschelt, I995), combinés avec des positions «welfare-chauvinistes » et, dans 
un certain nombre de cas, un discours anti-globalisation plus interventionniste et plus redistributif (Betz et Meret, 2013). Ces questions traversent également les travaux relatifs au vote radical populiste. Ces derniers suggèrent une moindre saillance des enjeux économiques pour les électeurs de ces partis (Oesch, 2008) et l'absence de positions clairement établies en la matière. Dans son analyse du cas belge, notamment, Derks (2006) illustre cette complexité en montrant la conjonction d'attitudes libérales, de soutien à l'État providence et de refus des politiques de redistribution de la gauche chez les électeurs de droite radicale. Il est à ce titre plus difficile de dégager un ensemble d'hypothèses quant au rôle des facteurs économiques dans le vote en faveur des droites radicales populistes.

\subsection{LA GAUCHE POPULISTE}

La formulation d'hypothèses pour la gauche populiste s'avère également plus délicate, compte tenu de l'absence relative de travaux comparatifs dans le contexte européen (Akkerman et Zaslove, 20I4). Comme nous l'avons noté plus haut, l'accent mis sur les enjeux socio-économiques constitue un facteur probable de différentiation, les partis de gauche populiste présentant un niveau plus élevé de saillance sur ces questions. En termes d'offre, les analyses de la gauche populiste ont montré l'importance pour ces mouvements de la régulation économique, de l'interventionnisme étatique ou de la redistribution des richesses, ainsi que leur opposition frontale au néolibéralisme (March, 2007 ; De Lange et Rooduijn, 20ıг ; Lucardie et Voerman, 20I2). De plus, on peut attendre de ces enjeux qu'ils soient intégrés dans un discours culturellement inclusif (Mudde et Rovira Kaltwasser, 20I3) : contrairement au «peuple» dans l'imaginaire de droite radicale, le peuple de la gauche populiste se présente sous la forme d'une entité plurielle, inclusive et politiquement active, non restreinte par des considérations de genre, d'origine, de race ou d'orientation sexuelle (Stavrakakis et Katsambekis, 20I4, p. I35).

La littérature sur le vote aboutit à des conclusions similaires. Les travaux sur les électeurs des gauches radicales - non nécessairement populistes par ailleurs - montrent que ces derniers soutiennent des politiques de régulation économique, qu’ils sont plus généralement en faveur de l'État provi- 
dence et particulièrement critiques des grandes sociétés multinationales (Visser et al., 20I4; Akkerman et Zaslove, 20I4).

$\mathrm{H}_{4}$. les électeurs de gauche populiste sont plus enclins à soutenir la régulation économique et la redistribution, et ils sont plus opposés à la globalisation que les électeurs mainstream.

S’agissant, en revanche, de la dimension culturelle, notamment des enjeux d’immigration et des valeurs autoritaires, les données comparatives disponibles sont sans doute plus éparses encore. Deux perspectives sont envisageables. Akkerman et Zaslove (20I4) illustrent la prééminence de l'appartenance de classe et de l'idéologie social-démocratique, qui favorisent selon eux une approche inclusive de l'immigration et conduisent à une reformulation des enjeux relatifs à la criminalité en termes strictement socio-économiques - milieu, classe, pauvreté. Par ailleurs, en ce qui concerne leur positionnement sur la dimension culturelle, nombre de formations de gauche radicale ont «voyagé » vers le pôle libertaire-cosmopolite (March, 20I I), ce qui les éloigne des préférences autoritaires de la droite radicale. À l'inverse, suivant une hypothèse de «compétition ethnique » (Rydgren, 2008), on peut envisager que l'électorat populaire des partis de gauche populiste perçoive l'immigration comme une source de concurrence sur le marché du travail, une crainte potentiellement renforcée par la crise économique, ce qui aurait pour effet d'accroître la saillance de ces enjeux et d'altérer le positionnement de ces électeurs sur l'axe culturel (Akkerman et Zaslove, 20I4). Les travaux existants tendent pour l'heure à infirmer cette seconde hypothèse (Visser et al., 20I4, Akkerman et Zaslove, 20I4).

$\mathrm{H}_{5}$. les électeurs de la gauche populiste ne sont pas significativement différents de ceux des partis mainstream au regard des attitudes nativistes et autoritaires. 


\subsection{INTÉGRATION EUROPÉENNE ET GLOBALISATION ÉCONOMIQUE}

Un des objectifs de cet article est de faire apparaître les liens entre populismes de gauche et de droite, d'une part, et intégration européenne, d’autre part. Le processus de construction européenne s'est imposé comme un enjeu dominant pour ce type de formations situées à la périphérie des systèmes partisans. Des études récentes ont noté que l'euroscepticisme représente un élément commun des populismes de gauche et de droite (De Vries et Edwards, 2009; Halikiopoulou et al., 20I2). Pour la droite radicale, cette hostilité à l'Europe recoupe plusieurs dimensions, depuis la défense de la souveraineté nationale, le rejet de l'immigration et de la libre circulation, et plus généralement la transposition au niveau européen de son discours anti-establishment traditionnel (Almeida, 20ı, p. 243). L'euroscepticisme représente par ailleurs un facteur explicatif significatif du vote pour ces partis (Werts et al., 20I3). Dans le cas des partis de gauche populiste, en revanche, l'antagonisme à l'encontre de l'Europe relève pour l'essentiel de l'euroscepticisme «soft» défini par Szczerbiak et Taggart (2008, vol. 2, p. 247-248) comme une opposition à la trajectoire, présente ou future, de l'UE et le refus de l'extension de ses compétences. En dépit de ces divergences, on peut anticiper:

H6. les électeurs des partis populistes de droite et de gauche présentent un degré d'euroscepticisme plus élevé que ceux des partis mainstream. L’effet de l'hostilité à l'Europe est plus important chez les supporters des partis de droite radicale.

Dans les deux cas, on note une forte hétérogénéité dans l'attitude de ces formations à l'égard de l'UE, à gauche (Charalambous, 20I I) mais aussi à droite. Y compris au sein de la famille de droite radicale, il existe une large gamme de positions vis-à-vis de la construction européenne, entre «rejet», «soutien conditionnel» et «recherche de compromis » (Vasilopoulou, 20II). On peut de ce fait entrevoir l'existence de variations importantes de nature et/ou d'intensité dans le rapport que les populistes entretiennent avec l'Europe et les motifs qui président à l'euroscepticisme exprimé par leurs supporters, autour notamment de la distinction entre «inclusivisme socio-économique» et «exclusivisme culturel». 
H7. les électeurs de droite radicale rejettent l'UE pour des motifs essentiellement culturels, liés notamment à l’immigration, tandis que les supporters de la gauche populiste s'opposent plus spécifiquement à ce qu’ils perçoivent comme une trajectoire néolibérale de l’intégration européenne.

Ces hypothèses s'inscrivent enfin plus largement dans un autre domaine de recherche, relatif à la transformation des clivages et au soutien pour les partis populistes. Kriesi et al. (2006, 2008) ont avancé la thèse d'un processus de «dénationalisation» articulant européanisation, immigration et globalisation économique, et ont suggéré l'émergence d'un nouvel axe de conflit structurel. Cet axe, indépendant du clivage gauche-droite, met en regard «gagnants » et «perdants» de la mondialisation, et vient s'intégrer dans la structure bidimensionnelle formée par les dimensions économique et culturelle. Autour d'une opposition entre «intégration» et «démarcation» (ibid., 2006, p. 922), les «perdants» adhèrent à un modèle protectionniste, replié sur l'espace national et la souveraineté, tandis que les «gagnants» voient dans le processus d'intégration internationale une opportunité. Jusqu’à présent, cet axe de conflit a été principalement rendu opératoire par les partis populistes de droite. Kriesi (2014, p. 369-370) suggère cependant que, dans le contexte actuel de la «grande» récession économique, les conséquences de la dénationalisation peuvent être mobilisées par de nouveaux challengers à gauche de l'axe politique, capables d'articuler les enjeux relatifs au processus d'intégration internationale en termes plus strictement socio-économiques. Hernandez et Kriesi (20I4, p. I I-I2) ajoutent à ce propos que la crise crée une structure d'opportunité favorable pour une gauche populiste capable d'adopter une position sociale et économique national-protectionniste.

H8. les électeurs des partis populistes se distinguent de ceux des autres partis par leur adhésion au protectionnisme économique et leurs attitudes négatives à l'égard de la globalisation. 


\section{DONNÉES, SÉLECTION DES CAS ET MÉTHODES}

Notre analyse s'appuie sur les données comparatives issues de l'enquête «Comparative Electoral Dynamics in the European Union in 20I4», conduite dans sept pays d'Europe occidentale . La variable dépendante est le vote déclaré aux élections européennes de 20I4. Les données de l'enquête permettent de localiser au total onze formations de gauche populiste et six partis appartenant à la droite populiste (voir le tableau i).

Dans l'espace européen, le populisme de gauche agrège un ensemble de formes idéologiques, de la gauche radicale au post-communisme, en passant par l'incorporation de valeurs libertaires post-matérialistes, féministes et écologistes. Ces différentes forces varient dans leur degré de populisme ainsi qu'au regard de leurs origines politiques (March, 20II). L'espace marxiste léniniste orthodoxe demeure aujourd'hui circonscrit à un petit nombre de partis qui, à l'image du KKE (Parti communiste grec) en Grèce, notamment, déploient la rhétorique populiste anti-européenne contre une «ploutocratie» rendue responsable de la crise. Des mouvements tels que L'Altra Europa con Tsipras en Italie, le Front de Gauche en France, la Izquierda Plural espagnole ou le Bloco de Esquerda portugais viennent de la gauche radicale traditionnelle. Ces partis ont toutefois progressivement évolué vers un modèle pragmatique de socialisme-populisme anti-establishment au travers de la réinterprétation du conflit historique de classes au prisme de la nouvelle dialectique peuple-élite évoquée précédemment. La composante populiste a également été repérée par la littérature dans des mouvements tels que SYRIZA d'Alexis Tsipras en Grèce (Stavrakakis et Katsambekis, 20I4) ou Die Linke en Allemagne (Decker, 2008). D’autres formations, enfin, à l'instar du $\mathrm{M}_{5} \mathrm{~S}$ en Italie ou de Podemos en Espagne ont émergé des mouvements sociaux (Alonso et Rovira Kaltwasser, 20I4; Corbetta et Gualmini, 20I3) ou sont le fruit d'une convergence avec les partis écologistes, telle la Coligação Democrática Unitária portugaise. Le positionnement systémique du populisme post-matérialiste incarné par le $\mathrm{M}_{5} \mathrm{~S}$ de Beppe Grillo

3 Enquête par Internet réalisée par TNS-SOFRES pour le Centre d'études européennes (CEE, Sciences Po), sous la direction de Nicolas Sauger, du 28 mai au 12 juin 2014, sur la France, l'Allemagne, l'Italie, l'Autriche, l'Espagne, la Grèce et le Portugal ( $N=22072)$. 
en particulier s'avère plus problématique et la spécificité de son soutien électoral est examinée en seconde partie de cet article.

L'identification des acteurs de la droite populiste répond quant à elle aux critères formulés par Mudde (2007), notamment pour des partis tels que le FN français, la Lega Nord italienne, le FPÖ autrichien ou le La.O.S. en Grèce, dont l'appartenance à la famille radicale populiste a été établie de longue date. Nous excluons en revanche des formations plus clairement situées à l'extrême-droite traditionnelle, notamment l'Aube dorée grecque ou les Fratelli d'Italia. S'agissant enfin de l'AfD allemande, il n'existe pas à ce jour de consensus au sein de la littérature. Arzheimer (2015) conclut à l'absence de marqueurs nativistes et populistes ainsi qu'à un euroscepticisme «soft » dans le programme du parti. Häusler (20I3) souligne en revanche la présence d'une composante de droite populiste aux côtés des tendances néolibérales et national-conservatrices. Berbuir et al. (20I4, p. 20) avancent un argument similaire au regard de l'hétérogénéité de la communication et de la stratégie de l'AfD, voyant dans le parti de Bernd Lucke un «équivalent fonctionnel» de la droite populiste européenne. Nous testons pour notre part le positionnement idéologique des électeurs de l'AfD dans une hypothèse de droite radicale populiste, hypothèse dont nous examinons la pertinence.

Les deux groupes d'acteurs populistes sont comparés, pour les besoins de l'analyse, avec les deux grandes familles mainstream de la droite conservatrice, libérale et chrétienne-démocrate, d'une part, et de la gauche social-démocrate, d'autre part, ainsi qu'avec les partis écologistes, qui représentent traditionnellement le pôle libertaire-cosmopolite de la dimension GAL/TAN (Green/ Alternative/Libertarian vs. Tradionalism/Authority/Nationalism, voir Hooghe et al., 2002). 
Tableau I. Partis populistes et mainstream aux élections européennes de 20I4*

\begin{tabular}{|c|c|c|c|c|c|}
\hline PAYS & $\begin{array}{l}\text { GAUCHE } \\
\text { POPULISTE }\end{array}$ & GAUCHE & ÉCOLOGISTES & $\begin{array}{c}\text { DROITE ET } \\
\text { CENTRE-DROIT }\end{array}$ & $\begin{array}{c}\text { DROITE } \\
\text { POPULISTE }\end{array}$ \\
\hline $\begin{array}{l}\text { FRANCE } \\
(40 \mid 3)\end{array}$ & $\begin{array}{l}\text { PG/PCF } \\
(139)\end{array}$ & $\begin{array}{c}\text { PS (362) } \\
\text { Autres (101) }\end{array}$ & $\begin{array}{c}\text { EELV (224) } \\
\text { Autres écologistes } \\
(45)\end{array}$ & $\begin{array}{l}\text { MoDem, UDI, } \\
\text { UMP (704) }\end{array}$ & $\mathrm{FN}(500)$ \\
\hline $\begin{array}{l}\text { ALLEMAGNE } \\
(40 \mid 4)\end{array}$ & $\begin{array}{l}\text { Die Linke } \\
\text { (328) }\end{array}$ & $\begin{array}{l}\text { SPD } \\
(626)\end{array}$ & $\begin{array}{l}\text { Grünen } \\
\text { (253) }\end{array}$ & $\begin{array}{c}\operatorname{FDP}(78) \\
\text { CDU/CSU (60I) }\end{array}$ & AfD (244) \\
\hline $\begin{array}{l}\text { ITALIE } \\
(4003)\end{array}$ & Altra Europa (I85) & $\begin{array}{l}\text { PD } \\
(10 \mid 0)\end{array}$ & $\begin{array}{l}\text { Green Italia } \\
\text { (37) }\end{array}$ & \begin{tabular}{|c|} 
Nuovo Centro \\
destra - Unione di \\
Centro (NCD- \\
UDC) (54) \\
Forza Italia (249) \\
Autres (54) \\
\end{tabular} & Lega Nord (I5I) \\
\hline $\begin{array}{l}\text { ESPAGNE } \\
(4010)\end{array}$ & $\begin{array}{c}\text { La Izquierda Plural } \\
\text { Podemos (486) }\end{array}$ & $\begin{array}{l}\text { PSOE } \\
(280)\end{array}$ & $\begin{array}{c}\text { Iniciativa per Cata- } \\
\text { lunya Verds (ICV) } \\
(180)\end{array}$ & \begin{tabular}{|c} 
Unión Progreso y \\
Democracia (UPD) \\
( I 75) \\
Partido Popular \\
(372)
\end{tabular} & \\
\hline $\begin{array}{l}\text { AUTRICHE } \\
(4002)\end{array}$ & $\begin{array}{c}\text { Europa Anders } \\
\text { (KPÖ) (73) }\end{array}$ & $\begin{array}{l}\text { SPÖ } \\
(342)\end{array}$ & $\begin{array}{l}\text { Grünen } \\
\text { (402) }\end{array}$ & $\begin{array}{c}\text { Das Neue Ös- } \\
\text { terreich (NEOS) } \\
(246) \\
\text { ÖVP (338) } \\
\text { Autres (26) }\end{array}$ & $\begin{array}{l}\text { FPÖ (4I I) } \\
\text { BZÖ (9) }\end{array}$ \\
\hline $\begin{array}{l}\text { GRĖCE } \\
(1017)\end{array}$ & $\begin{array}{c}\text { KKE (44) } \\
\text { SYRIZA (I8I) }\end{array}$ & $\begin{array}{l}\text { PASOK } \\
(20)\end{array}$ & $\begin{array}{l}\text { Oikologoi Prasinoi } \\
\text { (OP) (24) }\end{array}$ & $\begin{array}{c}\text { ND (82) } \\
\text { Autres (79) }\end{array}$ & La.O.S. (22)*** \\
\hline $\begin{array}{l}\text { PORTUGAL } \\
(10 \mid 3)\end{array}$ & \begin{tabular}{|c|} 
Coligação Demo- \\
crática Unitária \\
(CDU) (6I) \\
Bloco de Esquerda \\
(BE) (35)
\end{tabular} & $\begin{array}{l}\text { PS } \\
(84)\end{array}$ & $\begin{array}{c}\text { Partido Ecologista } \\
\text { "Os Verdes" (PEV) } \\
\text { (49) }\end{array}$ & $\begin{array}{l}\text { PSD } \\
(93)\end{array}$ & \\
\hline
\end{tabular}

*taille des échantillons d'électeurs entre parenthèses; ***exclu de l'analyse au regard de l'effectif trop faible; «autres partis» non spécifiés exclus de l'analyse. Partis non retenus: Anexartitoi Ellines (ANEL) en Grèce, Fratelli d'Italia - Alleanza Nazionale (Italie), XA (Aube dorée) en Grèce et Movimento 5 Stelle (M5S, $N=586$ ) en Italie (voir infra). 
Deux espaces d'analyse sont identifiés sur la base d'une distinction entre pays contributeurs au budget de l’UE et États membres bénéficiaires. Au sein de la zone euro, les déséquilibres structurels entre ces deux groupes de pays ont constitué un facteur déterminant de la crise des dettes souveraines (Lane, 2012). Pour ce qui concerne plus largement l'Europe occidentale, l'opportunité de cette différenciation entre créditeurs et débiteurs s'établit sur un ensemble de critères relatifs à l'impact de la crise économique et financière mais également à la structuration de la compétition partisane et aux niveaux différentiels de soutien électoral aux formations populistes (voir le tableau 2, ci-dessous).

Tableau 2. Compétition partisane, indicateurs socio-économiques et soutien aux partis populistes dans les États membres contributeurs et bénéficiaires (UE I5)

\begin{tabular}{|l|c|c|c|}
\hline ÉtATS MEMBRES & $\begin{array}{c}\text { SCORE MOYEN } \\
\text { GAUCHE POPULISTE } \\
\text { (ÉCART TYPE)* }\end{array}$ & $\begin{array}{c}\text { SCORE MOYEN } \\
\text { DROITE POPULISTE } \\
\text { (ÉCART TYPE)* }\end{array}$ & TAUX DE CHOMMAGE* \\
\hline BÉNÉFICIAIRES $(\mathrm{N}=5)$ & 19,2 & 3,8 & 17,3 \\
\hline CONTRIBUTEURS $(\mathrm{N}=10)$ & $(10,5)$ & $(5,3)$ & $(8,1)$ \\
\hline \multirow{2}{*}{ TOTAL UE I5 } & 5,7 & 16,1 & 7,6 \\
& $(4,0)$ & $(8,0)$ & $(2,4)$ \\
\hline
\end{tabular}

*différences significatives au seuil de $p<0,01$ (analyse de variance).

Les trois pays bénéficiaires présents dans l'enquête - Grèce, Espagne et Portugal - sont caractérisés par un niveau important de chômage, un soutien élevé pour les formations de gauche populiste ainsi que la faiblesse ou l'absence de partis de droite radicale, à l'exception d'Aube dorée en Grèce. Cette situation est proche de celle de certains pays d’Amérique latine durant les années 1990 et elle corrobore les hypothèses formulées par Kriesi et Pappas (20I4) quant à l'essor d'une gauche populiste dans les pays les plus sévèrement touchés par la crise. On note en outre la présence de gouvernements dirigés par des partis de droite pro-européens dans les trois cas $^{4}$, qui accroît la polarisation sur un axe

4 Partis et coalitions au pouvoir en mai 2014: France = PS, PRG (Gauche), Allemagne = CDU, CSU, SPD (Grande coalition), Italie = PD, NCD, SC, UdC (Grande coalition), 
pro/anti-austérité. Le spectre partisan est plus large en revanche dans les États membres contributeurs - France, Allemagne, Autriche et Italie -, où coexistent gauche et droite populistes. À l'exception du cas français, ces pays sont gouvernés par des grandes coalitions.

Les déterminants attitudinaux du soutien aux partis populistes de gauche et de droite sont examinés sur les principales dimensions idéologiques définies plus haut, à partir, lorsque les données le permettent, d'échelles cumulatives 5 . Une analyse en composantes principales (ACP), non détaillée ici, fait apparaître des corrélations significatives entre plusieurs groupes d'items, qui suggèrent la présence d'univers attitudinaux structurés. En lien avec nos hypothèses, quatre échelles sont construites pour le degré de satisfaction avec les institutions et élites politiques, le nativisme, la régulation et redistribution économiques ainsi que le soutien à l'intégration européenne ${ }^{6}$. Au regard des matrices de corrélations, quatre dimensions sont en revanche appréhendées à partir d'indicateurs simples : l'autoritarisme, le conservatisme moral, l'attitude à l'égard de la globalisation et le protectionnisme économique (voir le tableau 3, ci-après).

Autriche = SPÖ, ÖVP (Grande coalition), Espagne = PP (Droite), Grèce = ND (Droite), Portugal = PSD, CDS-PP (Droite).

5 Les échelles cumulatives permettent de réduire l'erreur aléatoire dans la mesure. Mclver and Carmines (198I, p. 15) soulignent à ce propos certaines faiblesses inhérentes aux mesures établies à partir d'items individuels, en termes de validité, de précision et de fiabilité.

6 Les scores sont obtenus par l'addition des réponses individuelles. La mesure de cohérence interne des échelles dérivées est satisfaisante mais demeure en-deçà toutefois du seuil généralement retenu (alpha $\geq 0,8$ ), à l'exception des deux questions relatives à l'immigration. Toutes les échelles et tous les indicateurs simples sont standardisés. 
Tableau 3. Indicateurs attitudinaux

\begin{tabular}{|c|c|c|c|}
\hline DIMENSION & INDICATEURS* & MOYENNE & ÉCART-TYPE \\
\hline \multirow{3}{*}{$\begin{array}{l}\text { SATISFACTION } \\
\text { INSTITUTIONS POLITIQUES } \\
(\text { alpha }=0,6)\end{array}$} & $\begin{array}{l}\text { Le Parlement français prend en compte les préoccu- } \\
\text { pations des citoyens. }\end{array}$ & 3,5 & 2,7 \\
\hline & $\begin{array}{l}\text { Approuvez-vous ou désapprouvez-vous le bilan du } \\
\text { Gouvernement français à ce jour? }\end{array}$ & 3,6 & 2,7 \\
\hline & $\begin{array}{l}\text { Les responsables politiques parlent trop et n'agissent } \\
\text { pas assez (inv.). }\end{array}$ & 1,6 & 2,2 \\
\hline \multirow{2}{*}{$\begin{array}{l}\text { NATIVISME } \\
(\text { alpha }=0,8)\end{array}$} & $\begin{array}{l}\text { L'immigration devrait être considérablement réduite } \\
\text { en France. }\end{array}$ & 6,4 & 3,1 \\
\hline & L'immigration menace nos emplois & 5,5 & 3,3 \\
\hline AUTORITARISME & $\begin{array}{l}\text { Les gens qui ne respectent pas la loi devraient être } \\
\text { punis plus sévèrement qu'aujourd'hui.. }\end{array}$ & 7,7 & 2,4 \\
\hline CONSERVATISME MORAL & $\begin{array}{l}\text { Le mariage ne devrait pas être autorisé pour les } \\
\text { couples de même sexe. }\end{array}$ & 3,5 & 3,7 \\
\hline \multirow{2}{*}{$\begin{array}{l}\text { RÉGULATION, } \\
\text { REDISTRIBUTION ÉCO. } \\
(\text { alpha }=0,6)\end{array}$} & $\begin{array}{l}\text { L'État devrait imposer plus de réglementations éco- } \\
\text { nomiques et contrôler le marché }\end{array}$ & 6,6 & 2,6 \\
\hline & $\begin{array}{l}\text { Les richesses devraient être plus redistribuées des } \\
\text { riches vers les pauvres. }\end{array}$ & 7,4 & 2,6 \\
\hline $\begin{array}{l}\text { GLOBALISATION } \\
\text { COMME OPPORTUNITÉ }\end{array}$ & $\begin{array}{l}\text { La globalisation est une chance pour la croissance } \\
\text { économique. }\end{array}$ & 5,4 & 2,6 \\
\hline \multirow{3}{*}{$\begin{array}{l}\text { SOUTIEN INTÉGRATION } \\
\text { EUROPÉENNE } \\
(\text { alpha }=0,7)\end{array}$} & $\begin{array}{l}\text { L'intégration européenne devrait être menée plus } \\
\text { loin. }\end{array}$ & 5,0 & 2,91 \\
\hline & $\begin{array}{l}\text { Vous faites confiance aux institutions de I'Union } \\
\text { européenne. }\end{array}$ & 4,1 & 2,7 \\
\hline & $\begin{array}{l}\text { La Commission européenne devrait avoir plus de } \\
\text { pouvoirs sur les politiques économiques et budgé- } \\
\text { taires des États. }\end{array}$ & 4,1 & 2,8 \\
\hline PROTECTIONNISME ÉCO. & $\begin{array}{l}\text { Des barrières douanières et le protectionnisme } \\
\text { économique devraient être rétablis. }\end{array}$ & 4,7 & 3,0 \\
\hline
\end{tabular}

*toutes variables ordinales, échelles 0-10; N=22072, listwise $N=20208$.

Un rapide examen des corrélations entre ces diverses dimensions montre la convergence du soutien à l’UE, de l'attitude à l'égard de la globalisation comme opportunité de croissance et du degré de satisfaction politico-institutionnelle, d'une part, et la corrélation attendue entre nativisme et autoritarisme, d’autre part. On note également une forte corrélation positive entre le nativisme et l'échelle de protectionnisme économique, qui corrobore les observations de Kriesi et al., (2006, 2008) 
quant à la structuration d'un axe de compétition «démarcation-intégration» articulé à la fois sur les dimensions économique et culturelle.

L'exploration des déterminants du vote en faveur des partis populistes de gauche et de droite est conduite au moyen de régressions logistiques multinomiales. Ces modèles sont appropriés pour l'analyse du vote (Dow et Endersby, 2004). Ils permettent «d'estimer simultanément les logits binaires pour toutes les comparaisons parmi les alternatives présentes» (Long et Freese, 2006, p. 224)7. L'effet des déterminants attitudinaux est contrôlé par les variables de genre, d'âge, de diplôme et de revenus, ainsi que par des variables dummy pour les pays. Les résultats des modèles de régressions logistiques multinomiales groupées pour les quatre pays contributeurs - France, Allemagne, Italie et Autriche - et les trois pays bénéficiaires - Grèce, Espagne et Portugal- sont présentés dans les tableaux A et A2 en annexes ${ }^{8}$. Dans le premier ensemble d'États membres, les formations populistes de gauche et de droite sont contrastées avec les principaux acteurs mainstream, mais également entre elles. Pour certains des indicateurs d'intérêt, nous présentons les résultats sous forme de graphiques de probabilités ajustées, qui aident à l'interprétation des effets principaux et des interactions (Mood, 20ı), s'agissant notamment ici de certaines variations observables au plan national.

7 Ces modèles présentent en outre l'avantage ne faire aucune hypothèse de normalité, linéarité et homogénéité de variance pour les variables indépendantes.

8 Les annexes sont accessibles en ligne à l'adresse suivante: <http://ress.revues.org/3009>. Outre les données liées à la situation politique et la conjoncture spécifique de ces deux ensembles, le choix d'introduire la dimension nationale sous forme de variables dummy pour les pays est motivé par l'existence de corrélations significatives entre les paramètres du modèle et les pays, et ce à l'intérieur de chacun des deux groupes. Cette procédure permet de contrôler l'hétérogénéité non observée qui pourrait être attribuable à ces variations nationales. 


\section{RÉSULTATS}

Pour ce qui concerne en premier lieu les quatre pays bailleurs de fonds de l'UE - France, Allemagne, Italie et Autriche -, les paramètres généraux du modèle attestent de la présence d'associations statistiques avec la variable dépendante de vote $^{9}$. Des effets significatifs sont observables pour l'ensemble des dimensions idéologiques ainsi que pour les contrôles sociodémographiques. L’analyse est cependant restreinte aux facteurs attitudinaux.

\section{I.LA DROITE RADICALE POPULISTE}

Les variables culturelles - immigration, autoritarisme et conservatisme moral - se révèlent des prédicteurs importants du vote en faveur de la droite radicale populiste, en accord avec la littérature. Conformément à nos anticipations ( $\mathrm{H}_{2}$ ), le nativisme isole clairement les supporters des droites populistes de l'ensemble des autres partis, gauche et droite confondues. Ces électeurs se distinguent des formations de gauche par leur degré d’autoritarisme et de conservatisme social, qui les rapproche à l'inverse des partis de la droite classique, une proximité qui réfute partiellement notre hypothèse H3. Une même conclusion peut être établie quant à l'effet du rejet de l'intégration européenne, qui contribue clairement à différencier la droite radicale dans ces quatre pays, à la fois des acteurs plus mainstream mais également de leurs compétiteurs populistes à gauche de l'échiquier politique, conformément à notre hypothèse initiale (H6).

Les variables économiques font apparaître une combinaison de protectionnisme et d'opposition à la globalisation, et une position «médiane» sur la variable de redistribution. Le protectionnisme économique favorise dans tous les cas de figure de manière significative le vote en faveur des droites radicales populistes, y compris vis-à-vis des partis de gauche populiste. L’attitude négative à l'égard de la globalisation économique montre en revanche une convergence de la droite

9 Tous les tests de ratio de vraisemblance sont significatifs à $p<0,001$. Le modèle final présente une diminution significative du log-vraisemblance $\left(C h{ }^{2}=49 \mid 8,6 d l=60 p<0,00 I\right)$, un pseudo $R^{2}$ égal à 0,5 (Nagelkerke) et un taux de succès classificatoire de 56,4\%. Ce taux est supérieur au critère généralement retenu d'une amélioration de $25 \%$ du taux proportionnel de précision aléatoire et du critère de chance maximum, qui prennent respectivement ici les valeurs de 34,3 et $46,2 \%$. 
populiste avec l'ensemble des mouvements de gauche, en opposition aux électeurs des partis de droite et du centre qui demeurent quant à eux plus positifs quant aux opportunités offertes par la mondialisation. Ces résultats attestent de l'existence d'un axe de compétition structuré à la fois par les enjeux économiques et culturels du processus de dénationalisation, dont le pôle de «démarcation» est largement préempté par les partis de la droite populiste. Enfin, les électeurs de ces partis occupent une position intermédiaire entre les blocs de gauche et de droite sur la dimension de redistribution et de régulation économiques, plus proches cependant des partis de centre-droit sur ces questions.

\subsection{LE POPULISME COMME UNIFICATEUR}

Conformément à notre hypothèse de départ $\left(\mathrm{H}_{\mathrm{I}}\right)$, une convergence est visible entre partis populistes de gauche et de droite sur la dimension politicoinstitutionnelle, dont on rappelle qu'elle constitue ici notre variable proxy pour la mesure des attitudes populistes. Les électeurs de ces formations partagent une même insatisfaction vis-à-vis des institutions politiques et des élites, un mécontentement à l'égard de l'action du gouvernement, qui les opposent à l'ensemble des partis mainstream - gauche social-démocrate, écologistes, centre et droite classique. Populistes de gauche et de droite adhèrent par ailleurs à l'idée d'une coupure entre les élites et le peuple, telle quapproximée dans la constitution de la variable attitudinale. L'effet de cette défiance institutionnelle demeure un facteur déterminant du vote en faveur des partis de gauche populiste, qui différencie notamment les électeurs de mouvements tels que Die Linke en Allemagne, l'Altra Europa en Italie ou le Front de Gauche français de leurs homologues sociaux-démocrates et écologistes.

Les variations nationales sont examinées dans les graphiques d'effets ci-après (voir Figure I). Dans tous les cas, la défiance accroît de manière substantielle la probabilité du vote populiste. En France, la coïncidence des courbes illustre la propriété conjointe des populismes hexagonaux. Les résultats montrent en revanche un effet de plus grande magnitude pour la gauche populiste en Allemagne et en Italie, éclairant pour partie la nature plus «institutionnelle » du populisme droitier dans ces deux contextes, notamment pour 
ce qui concerne la Lega Nord après sa participation aux divers gouvernements de centre-droit en Italie depuis le milieu des années 1990.

Figure I. Probabilités de vote pour les partis populistes de gauche et de droite en fonction du degré de satisfaction avec les institutions et les élites politiques
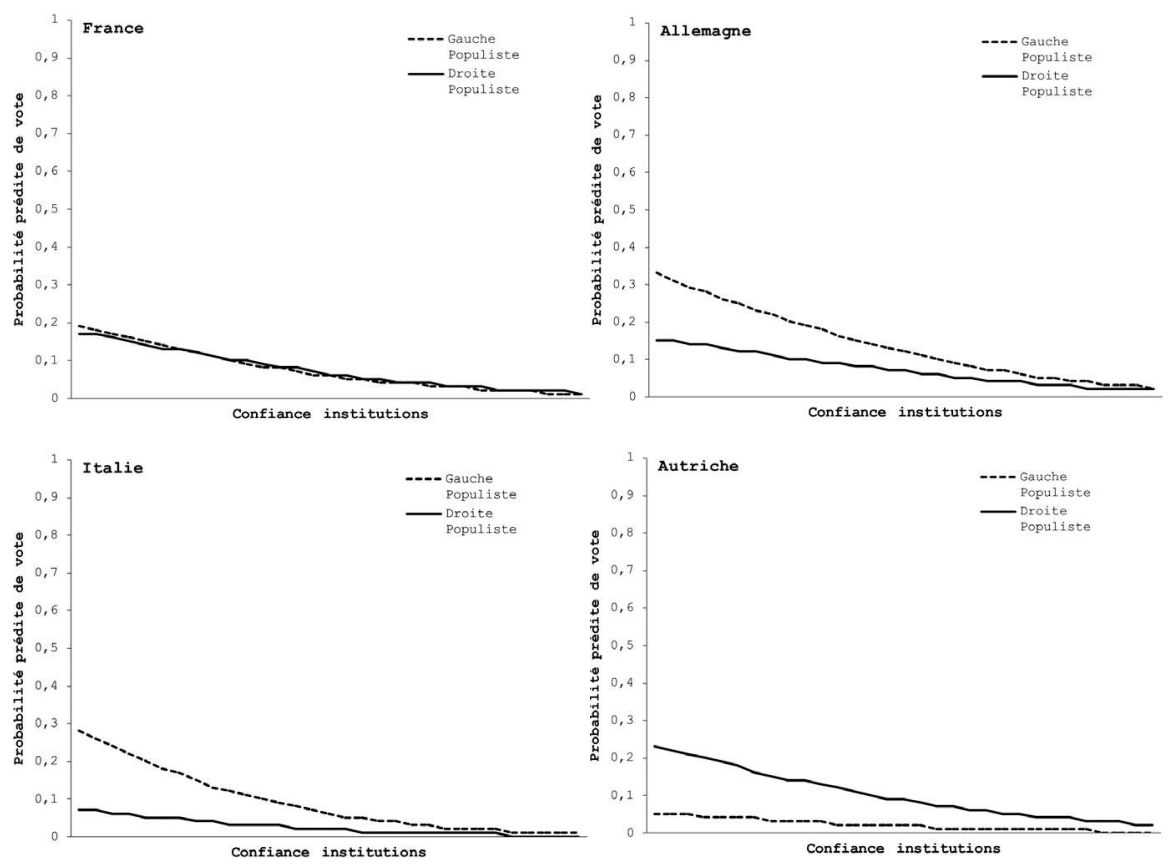

Note: graphes de probabilités ajustées pour l'effet de la variable «satisfaction avec les institutions et élites politiques», pour chacun des quatre pays contributeurs, en maintenant toutes les autres variables à leur moyenne d'échantillon; variable qualitative de genre fixée à modalité de référence 0 «Homme».

S'agissant de l'AfD, outre-Rhin, les résultats confirment la convergence attitudinale de son pool d'électeurs avec le modèle général de vote en faveur de la droite radicale paneuropéenne. L’AfD apparaît clairement ancrée à droite du système partisan allemand sur les questions économiques et les dimensions d'autoritarisme et de conservatisme social à l'endroit desquelles ses électeurs ne diffèrent pas significativement de ceux de la CDU/CSU et du FDP. La convergence 
avec le modèle général de droite radicale se fait sur trois dimensions clés que sont le nativisme, la défiance politico-institutionnelle et l'euroscepticisme. Dans tous les cas de figure, le nativisme augmente de façon substantielle la probabilité de vote pour l'AfD, de même que l'hostilité à l'intégration européenne et le degré d'insatisfaction mesuré à l'encontre des institutions et des élites politiques, cette dernière caractéristique étant commune aux populistes de gauche de Die Linke.

\subsection{LA GAUCHE POPULISTE}

Si nous nous tournons à présent vers la gauche de l'axe politique, en termes économiques, les résultats sont conformes à nos anticipations $\left(\mathrm{H}_{4}\right)$ : la critique du libéralisme, telle qu'elle s'exprime par la demande de politiques de redistribution et de régulation économique, accroît de manière significative la probabilité de voter pour les mouvements de la gauche populiste, de même qu'une attitude négative à l'égard de la globalisation. En revanche, l'adhésion au protectionnisme économique n’a pas d'effet significatif sur le vote en faveur de la gauche populiste dans l'espace des pays contributeurs, contrairement à ce qui a pu être observé plus haut pour les droites radicales dont le soutien croît avec le niveau de protectionnisme, ce qui infirme en partie notre hypothèse initiale (H8).

Sur la dimension culturelle, également, les résultats sont contraires à certaines de nos attentes $\left(\mathrm{H}_{5}\right)$ : les supporters des gauches populistes montrent un moindre degré de nativisme et d'autoritarisme par rapport aux électeurs des partis de gauche mainstream. Cette ouverture les rapproche des partis écologistes situés au pôle libertaire-cosmopolite de cet axe de compétition, et elle témoigne, conformément ici à notre anticipation ( $\mathrm{H} 7)$, d'un framing inclusif et pour l'essentiel socio-économique des questions relatives à l'immigration ou à la criminalité, diamétralement opposé à celui des droites radicales. Ces observations rappellent que beaucoup des partis de gauche radicale ont par ailleurs très largement incorporé les enjeux post-matérialistes au sein de leurs corpus doctrinal (March, 20II).

Enfin, les résultats corroborent l'hypothèse d'un euroscepticisme «soft» au sein des gauches populistes allemande, française, italienne et autrichienne (H6). La magnitude des effets de la critique de l'UE demeure très faible ainsi qu'illustrée par les probabilités du vote populiste de gauche, ajustées en fonc- 
tion de niveau d'euroscepticisme. Par contraste, l'élévation du niveau d'adhésion à la construction européenne diminue de manière substantielle et significative la propension au vote en faveur des partis de droite radicale populiste, notamment en France, en Allemagne et en Autriche (voir la figure 2).

Figure 2. Probabilités de vote pour les partis populistes de gauche et de droite en fonction du degré de soutien à l'intégration européenne
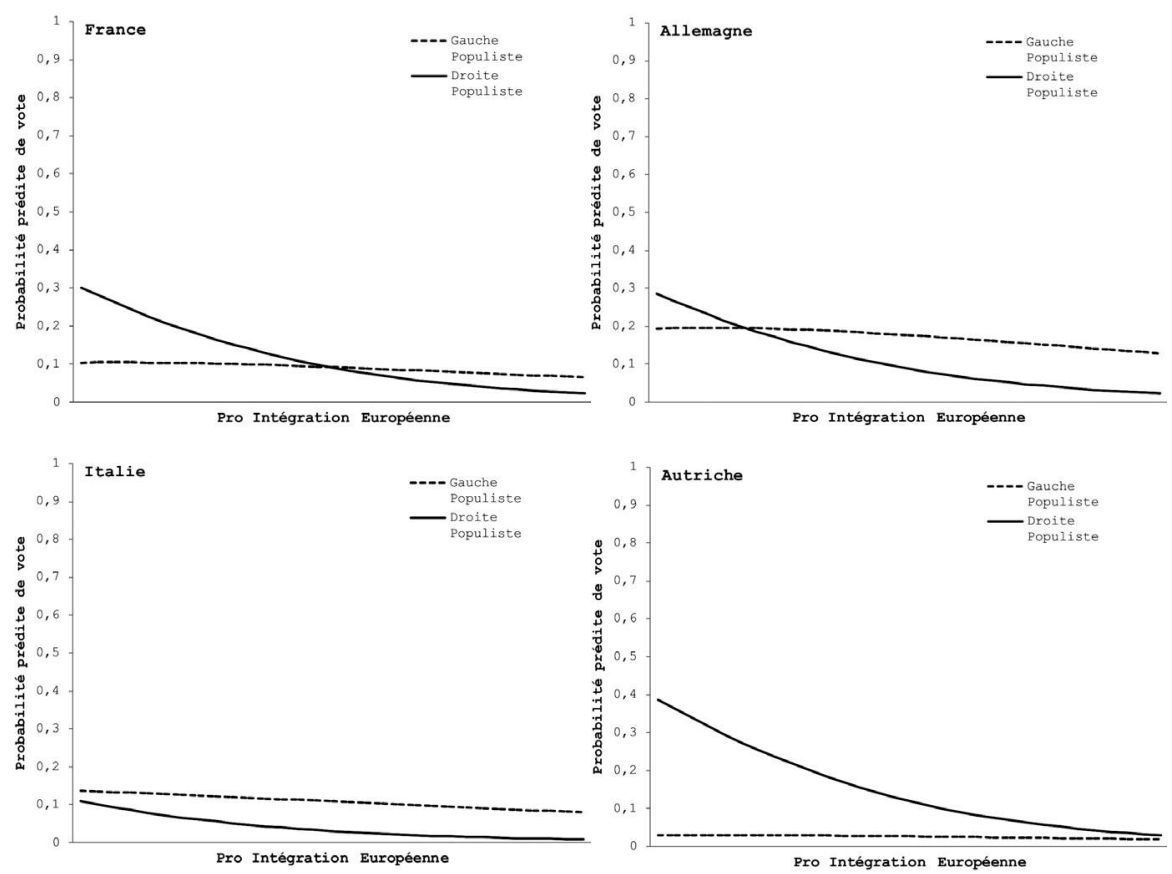

Note: graphes de probabilités ajustées pour l'effet de la variable «soutien intégration européenne», pour chacun des quatre pays contributeurs, en maintenant toutes les autres variables à leur moyenne d'échantillon; variable qualitative de genre fixée à modalité de référence 0 «Homme».

Ce résultat, important, est conforme à nos attentes de départ $(\mathrm{H} 6, \mathrm{H} 7)$ et confirme plus largement les principales hypothèses formulées dans la littérature sur l'euroscepticisme. Kriesi et al. (2008) suggèrent, rappelons-le, un lien entre le soutien à l'intégration européenne et la présence d’attitudes cosmopolite, 
impliquant que l'adhésion au projet de construction européenne est principalement corrélée avec les enjeux culturels. Ici, le constat d'un effet plus modéré de l'euroscepticisme chez les supporters des gauches populistes, lorsque comparés aux électeurs des droites radicales, est conjoint à l'observation d’attitudes plus tolérantes en matière d'immigration, d'autoritarisme ou de conservatisme moral.

\subsection{AU SUD DE L'EUROPE...}

Nous observons des distinctions importantes concernant notamment les variables politico-institutionnelles lorsque nous nous tournons vers les pays bénéficiaires du sud de l'Europe - Espagne, Grèce et Portugal. Les résultats révèlent une structure de compétition spécifique dans les trois États «débiteurs » de l'UE où les systèmes partisans sont plus fortement régis par l'opposition entre le pôle conservateur, d'une part, et la gauche populiste, d'autre part. C'est notamment le cas en Espagne où le bloc populiste (Izquierda Plural et Podemos) est venu concurrencer fortement le PSOE (Parti socialiste ouvrier espagnol) en mai 2014 et, naturellement, en Grèce avec la quasi-disparition du PASOK (Mouvement socialiste panhellénique) sous l'impact de SYRIZA. Il faut souligner à cet égard l'ampleur de la reconfiguration du jeu partisan en Grèce lors des législatives de mai 20I2, où les partis de gouvernement - PASOK et Nouvelle Démocratie - ont enregistré une perte de près de la moitié de leurs votes de 2009.

Pour ce second modèle, des associations statistiques significatives sont observables pour l'ensemble des variables indépendantes, à l'exception du genre $^{10}$; les dummies pour les divers pays sont également significatives indiquant des variations par rapport à la modalité de référence (Grèce) (voir le tableau A2 en annexes ${ }^{11}$ ). Les effets des principaux indicateurs d'intérêt sont détaillés au plan national dans les graphes de probabilités ajustées, présentés dans la figure 3.

I0 À l'exception du genre, le vote est statistiquement associé à l'ensemble des variables explicatives (tests de ratio de vraisemblance, $p<0,05$ ). Une diminution significative du log-vraisemblance $\left(\mathrm{Chi}^{2}=\mid 405,55 \mathrm{dl}=42 \mathrm{p}<0,00 \mathrm{l}\right.$ ) est observable dans le modèle final, avec un pseudo $\mathrm{R}^{2}$ égal à 0,5 (Nagelkerke) et un taux de succès classificatoire de $64,8 \%$ (contre 38,2 et 52,8\% respectivement pour le taux proportionnel de précision aléatoire et le critère de chance maximum, augmentés chacun de $25 \%$ ).

II <http://ress.revues.org/3009>. 
Figure 3. Probabilités de vote pour les partis populistes de gauche en Espagne, en Grèce et au Portugal
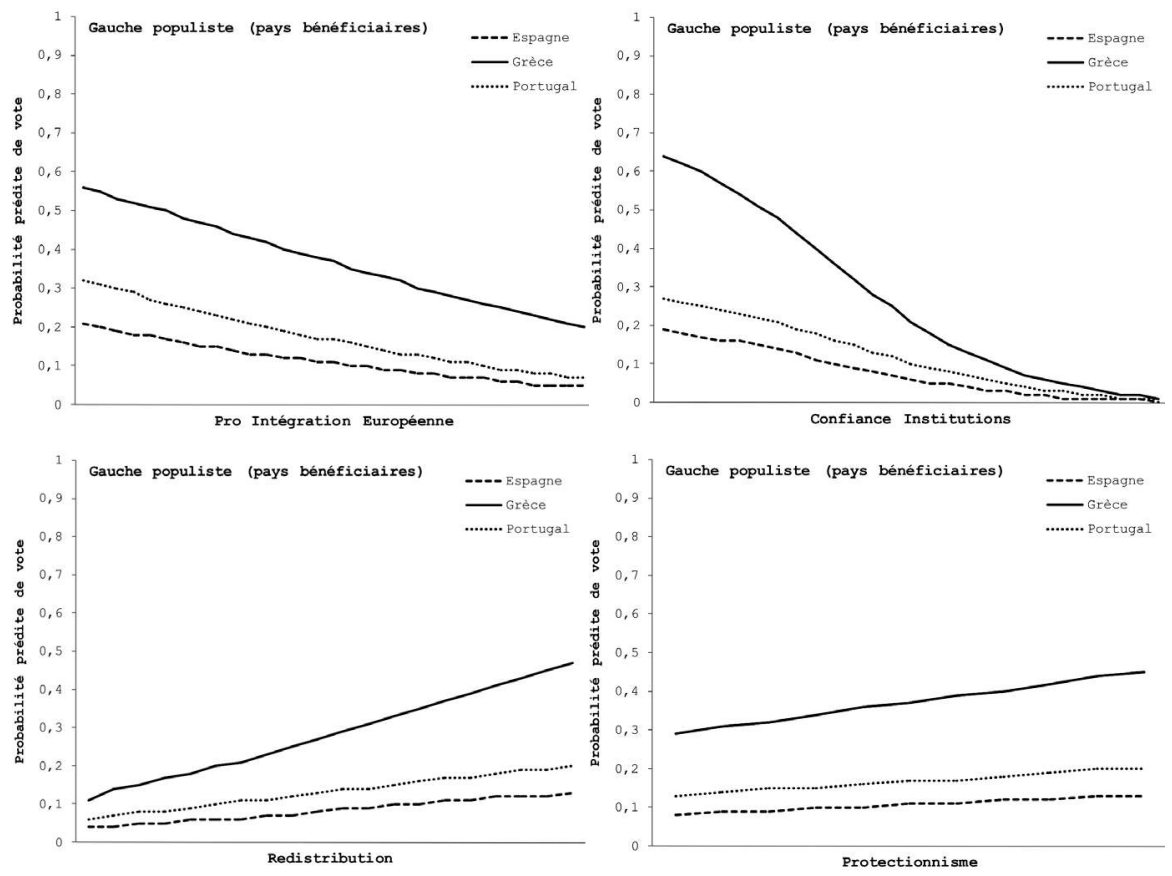

Note: graphes de probabilités ajustées pour l'effet des variables «soutien intégration européenne», «satisfaction institutions politiques», «régulation, redistribution» et «protectionnisme économique» pour chacun des trois pays bénéficiaires, en maintenant toutes les autres variables à leur moyenne d'échantillon; variable qualitative de genre fixée à modalité de référence 0 «Homme».

Sur l'ensemble des trois pays, le rapport aux institutions politiques et la perception des élites - nationales et européennes - demeurent des facteurs déterminants du vote en faveur de la gauche populiste, conformément à nos anticipations (Hı, H6). L'euroscepticisme accroît de manière significative la propension à soutenir ces formations et marque notamment une césure nette au sein des gauches en Europe du sud, entre socialistes mainstream et populistes. Une polarisation attitudinale plus nette encore est observable dans les paires «gauche populiste-droite classique» sur la dimension d'insatisfaction politico-institutionnelle, 
qui mêle, il faut le rappeler, la critique de l'action du gouvernement, du parlement et des élites partisanes ${ }^{12}$. Ces résultats corroborent nos hypothèses en même temps qu'ils éclairent la stratégie de partis tels que Podemos ou SYRIZA dont l'objectif est précisément d’articuler le rejet des élites nationales avec la crise de l'UE. Dinar et Rori (20I3, p. 274-276) soulignent à ce propos l'émergence en Grèce d'un nouvel axe de conflit opposant supporters et adversaires des plans d'aide. C'est en Grèce que la magnitude de l'effet de la variable politico-institutionnelle est la plus forte, avec une diminution sensible de la probabilité de vote pour SYRIZA à mesure que croît le niveau de satisfaction politique (voir la figure 3).

L'effet des variables politico-institutionnelles nationales et européennes contraste avec celui des facteurs économiques dont l'impact demeure en définitive beaucoup plus limité. À l'exception de la Grèce, la demande de redistribution et de régulation étatique n'augmente que plus marginalement le soutien aux formations de gauche populiste, donnant un soutien partiel à notre hypothèse de départ $\left(\mathrm{H}_{4}\right)$. De manière plus importante encore, on remarquera l'absence d'effet significatif de la variable de protectionnisme économique, qui imprègne indifféremment les électorats de la droite de gouvernement et de la gauche populiste. Ce résultat réfute notre hypothèse (H8) et l'argument avancé par Hernandez et Kriesi (20I4) d'une structure d'opportunité favorable à l'élaboration d'un programme de protectionnisme socio-économique par les gauches populistes dans les pays les plus sévèrement touchés par la crise.

\subsection{BEPPE GRILLO ET LE M5S}

Un dernier ensemble de questions concerne la nature du populisme post-matérialiste incarné par le $\mathrm{M}_{5} \mathrm{~S}$ en Italie depuis 20ı. Le cas atypique de la formation de Beppe Grillo est particulièrement intéressant, s'agissant de son placement sur l'axe gauche-droite, de son offre programmatique et plus encore des motivations de ses électeurs. Le message politique du mouvement combine une position libertaire de gauche - principalement sur les enjeux

12 Rappelons également que dans les trois pays concernés ici, le gouvernement est occupé au moment de l'enquête par les partis de droite, qui incarnent le pôle institutionnel pro-européen promoteur des politiques d'austérité. 
écologistes - avec des éléments droitiers, notamment un relatif conservatisme culturel et des politiques qui pourraient être interprétées comme anti-immigration. Au plan électoral, le $\mathrm{M}_{5} \mathrm{~S}$ mobilise des électeurs d'extrême-gauche, de gauche modérée, mais également à droite ainsi quauprès d’anciens abstentionnistes (Corbetta et Vignati, 20ı3 ; Bordignon et Ceccarini, 20ı3).

La stratégie retenue ici pour l'examen des déterminants attitudinaux du vote en faveur du $\mathrm{M}_{5} \mathrm{~S}$ est de conduire une analyse distincte contrastant les supporters de Beppe Grillo avec l'ensemble des autres forces politiques italiennes, y compris l'Altra Europa à gauche et la Lega Nord à droite. Les résultats de la régression logistique multinomiale sont présentés dans le tableau $\mathrm{A}_{3}$ en annexes ${ }^{13}$. Le $\mathrm{M}_{5} \mathrm{~S}$ y est défini comme modalité de référence; les paramètres généraux du modèle attestent de la présence d'associations statistiques avec la variable dépendante de vote ${ }^{14}$.

Les résultats confirment que le $\mathrm{M}_{5} \mathrm{~S}$ est clairement ancré à gauche de l'axe politique: ses électeurs ne se distinguent pas des autres formations de gauche sur les dimensions de redistribution, de globalisation et de protectionnisme ${ }^{15}$. Culturellement, ils ressemblent à leurs homologues de gauche populiste et écologistes, à l'exception du conservatisme moral où ils sont plus proches des électeurs du Partito Democratico (PD) de Matteo Renzi. Comparé à la gauche populiste des autres pays d'Europe du sud, l'effet de l'euroscepticisme demeure ici plus limité et n’accroît que de manière plus faible la probabilité de vote en faveur du mouvement de Beppe Grillo (voir la figure 4), reflétant sans doute pour partie l'ouverture culturelle dont font montre ses électeurs. Le rapport aux institutions et aux élites politiques constitue en revanche un facteur déterminant du vote $\mathrm{M}_{5} \mathrm{~S}$, qui différencie les «grillistes» de l'ensemble des autres électeurs, y compris les soutiens d'Altra Europa et de la Lega Nord.

Voir <http://ress.revues.org/3009>.

14 Tests de ratio de vraisemblance significatifs à $p<0,00$ | pour l'ensemble des variables attitudinales et l'âge, non significatifs pour le niveau de diplôme, le revenu et le genre. Le modèle final présente une diminution significative du log-vraisemblance $\left(\mathrm{Chi}^{2}=|| 8|, 8 \mathrm{dl}=60 \mathrm{p}<0,00|\right)$, un pseudo R² égal à 0,45 (Nagelkerke) et un taux de succès classificatoire satisfaisant de $55,9 \%$.

15 Un examen des classes d'affectation prédites par le modèle logistique montre que $36 \%$ des électeurs du M5S sont affectés au groupe de «gauche» mainstream, représenté par le PD, contre $49 \%$ dont le vote est anticipé correctement par le modèle. 
Figure 4. Probabilités de vote pour les partis populistes en Italie
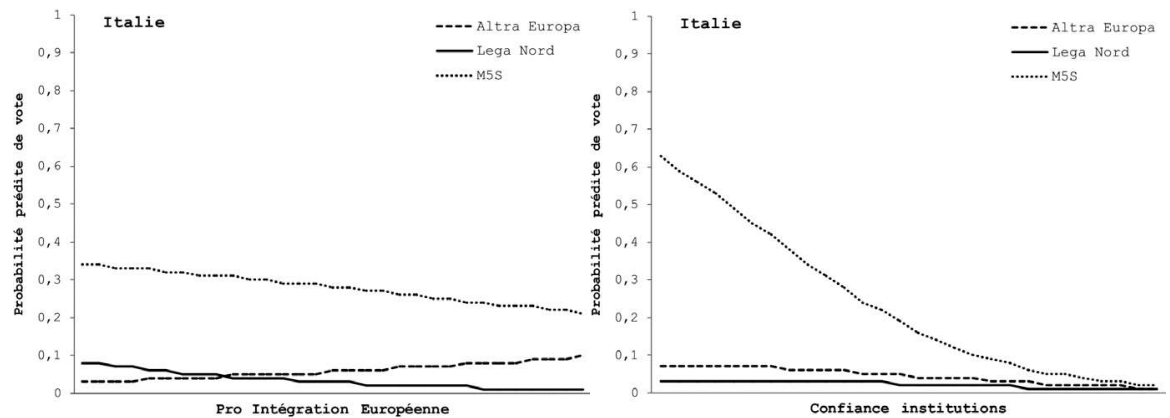

Note: graphes de probabilités ajustées pour l'effet des variables «soutien intégration européenne» et «satisfaction institutions politiques» pour l'Altra Europa, la Lega Nord et le Movimento 5 Stelle, en maintenant toutes les autres variables à leur moyenne d'échantillon (Base=ltalie); variable qualitative de genre fixée à modalité de référence 0 «Homme».

\section{CONCLUSION}

Cet examen des populismes nous a livré plusieurs enseignements quant aux développements récents du phénomène et aux demandes électorales pour ce type de partis en Europe occidentale. Les résultats attestent en premier lieu de l'importance du rapport à la sphère politico-institutionnelle dans la dynamique populiste et de la mobilisation différentielle que ces partis opèrent auprès des wutbürger détournés des institutions et les plus critiques à l'égard des élites politiques. Cette mécanique est à l'œuvre de manière particulièrement saillante dans les pays bénéficiaires du sud de l’Europe, là où le lien entre crise économique, austérité et échec des partis mainstream est le plus visible et où ces éléments s’articulent avec la critique de l’UE.

La question de la complexité de la relation entre populisme et euroscepticisme est clairement posée. Les perspectives vis-à-vis de l'intégration européenne varient au sein de la constellation populiste et dans l'espace européen. L'évaluation négative de l'UE, de ses institutions et de son rôle économique est au cœur de la dynamique électorale de formations telles que SYRIZA en 
Grèce ou Podemos en Espagne, articulée au rejet des élites nationales traditionnelles. Dans les pays contributeurs de l'UE, en revanche, l'impact du sentiment anti-européen se manifeste de manière sans doute moins nette qu'il n'est souvent anticipé dans la littérature. L'effet de l'euroscepticisme y demeure plus marginal pour les formations populistes de gauche alors qu'il joue un rôle central dans la mobilisation par les partis populistes de droite. Comme nous l'avons noté, ce poids différentiel tient pour beaucoup à la dimension culturelle à laquelle se rattache l'hostilité affichée notamment par les droites radicales à l'encontre du projet européen.

Le positionnement sur l'axe culturel opère une séparation nette entre populismes de gauche et de droite dans le contexte européen. Chez les premiers, l'adhésion à des valeurs d'ouverture plus libertaires et cosmopolites s'exprime de manière indifférenciée au nord et au sud de l'Europe. Elle tranche avec le noyau nativiste autoritaire des droites radicales populistes en France, en Italie ou en Autriche, dont nos résultats suggèrent qu'il structure également de manière significative le vote en faveur de l'AfD en Allemagne et dont il faudra à l'avenir mesurer comparativement les effets auprès d'autres formations de droite populiste, telles que l'UKIP au Royaume-Uni ou le Parti des Finnois de Timo Soini en Finlande.

En regard, l'axe traditionnel de conflit économique apparaît comparativement moins déterminant pour les populismes européens, notamment dans les pays bénéficiaires de l'UE où domine la défiance à l'encontre des élites politiques nationales et européennes. Sans surprise, les électorats des formations de gauche populiste témoignent d'un soutien plus marqué aux politiques de redistribution et d'une perception négative de la globalisation. Cette structure attitudinale les place à gauche sur la dimension économique. Les droites radicales populistes occupent quant à elles une situation intermédiaire sur l'axe de redistribution, entre blocs de gauche et de droite mainstream. Cette position illustre la plus faible saillance et la variabilité socio-économique parmi des partis tels que le FN français, l’AfD en Allemagne, le FPÖ autrichien ou la Lega Nord transalpine, en termes d'offre programmatique ainsi que de demande électorale. Pour ces mouvements, les questions économiques s'alignent en partie sur la 
dimension culturelle, ainsi qu'en témoignent l'impact de la perception négative de la globalisation et leur adhésion au protectionnisme dont on a souligné l'interconnexion avec le repli culturel nativiste.

En définitive, l’analyse de la matérialisation du phénomène populiste lors des élections européennes de 20I4 montre l'existence de modèles distincts de populisme de gauche et de droite, dont le dénominateur commun demeure le rapport que les citoyens entretiennent avec leur système politique et leurs élites partisanes. En suggérant une différenciation entre pays bénéficiaires et bailleurs de fonds de l'UE, nos résultats éclairent également la présence de contextes socio-politiques spécifiques, en lien avec les conjonctures économiques et la reconfiguration des systèmes de partis notamment au sud de l'Europe. L'essor d'un phénomène tel que le $\mathrm{M}_{5} \mathrm{~S}$ est à ce titre particulièrement intéressant, qui place, nous l'avons souligné, l'Italie à mi-chemin entre les pays «riches» du Nord et ceux qui, au Sud, ont senti plus quailleurs le poids de l'austérité. Au-delà de cette diversité cependant, nos résultats suggèrent l'existence de lignes de clivages transversales dans l'espace européen, structurées par l'impact différentiel de la crise économique et la gestion de cette dernière par l'UE et les élites nationales.

\section{BIBLIOGRAPHIE}

ADORNO Th. et al., 1950, The Authoritarian Personality, New York, Harper.

AKKERMAN A., MUDDE C. et ZASLOVE A., 2013, «How Populist Are the People? Measuring Populist Attitudes in Voters», Comparative Political Studies, 47-9, p. 1324-1353.

AKKERMAN A. et ZASLOVE A., 2014, «"We the People" or "We the Peoples"? A Comparison of Left- and Right-Wing Populists», ECPR Glasgow, 3-6 September.

ALONSO S. et ROVIRA KALTWASSER C. R., 20I4, «Spain: No Country for the Populist Radical Right?», South European Society and Politics, [en prepublication en ligne]: <doi:I0.1080/I3608746.20|4.985448>. 
ARZHEIMER K., 2015, «The AfD: Finally a Successful Right-Wing Populist Eurosceptic Party for Germany?», West European Politics, 38, [à paraître].

BERBUIR N., LEWANDOWSKYM. et SIRI J., 20I4, «The AfD and its

Sympathisers: Finally a Right-Wing Populist Movement in Germany?», German Politics, [en prepublication en ligne]: <doi:10.1080/09644008.2014.982546>.

BETZ H. G. et JOHNSON C., 2004, «Against the Current-Stemming the Tide: the Nostalgic Ideology of the Contemporary Radical Populist Right», Journal of Political Ideologies, 9-3, p. 311-327.

BETZ H.-G. et MERET S., 2013, «Right-Wing Populist Parties and the WorkingClass Vote. What Have You Done for Us Lately? », in Class Politics and the Radical Right, J. Rydgren (éd.), London and New York, Routledge, p. I07-121.

BORDIGNON F. et CECCARINI L., 2013, «"Tsunami” a 5 stelle», in Un salto nel voto. Ritratto politico dell'Italia di oggi, Ilvo Diamanti (éd.), Bari, Editori Laterza, p.60-7I.

CANOVAN M., 1999, «Trust the People! Populism and the Two Faces of Democracy», Political Studies, 47-I, p.2-16.

CHARALAMBOUS G., 20II, «All the Shades of Red: Examining the Radical Left's Euroscepticism », Contemporary Politics, 17-3, p. 299-320.

CORBETTA P. et VIGNATI R., 2013, «Left or Right? The Complex Nature and Uncertain Future of the 5 Stars Movement», Italian Politics and Society: The Review of the Conference Group on Italian Politics and Soceity, 72-73, p. 53-62.

CORBETTA P. et GUALMINI E. (éds), 2013, II partito di Grillo, Bologna, II Mulino.

DECKER F., 2008, «Germany: Right-wing populist failures and left-wing successes», Twenty-First Century Populism, New York, Palgrave Macmillan, p. I19-134.

DE LANGE S. et ROODUIJN M., 20II, «Een populistische Zeitgeist in Nederland? Een inhoudsanalyse van de verkiezingsprogramma's van populistische en gevestigde politieke partijen », in Democratie Doorgelicht, het functioneren van de Nederlandse democratie, R. Andeweg et J. Thomassen (éds), Leiden, Leiden University Press, p. 319-348. 
DERKS A., 2006, «Populism and the Ambivalence of Egalitarianism. How Do the Underprivileged Reconcile a Right Wing Party Preference with Their SocioEconomic Attitudes?», World Political Science Review, 2-3, p. 175-200.

DE VRIES C.E. et EDWARDS E. E., 2009, «Taking Europe to Its Extremes: Extremist Parties and Public Euroskepticism», Party Politics, I5-I, p. 5-28.

DINAR E. et RORI L., 2013, «The 2012 Greek Parliamentary Elections: Fear and Loathing in the Polls», West European Politics, 36-I, p. 270-282.

DOW J.K. et ENDERSBY J.W., 2004, «Multinomial Probit and Multinomial logit: A Comparison of Choice Models for Voting Research», Electoral Studies, 23, p. 107-122.

DUNN K., 2013, «Preference for Radical Right-Wing Populist Parties Among Exclusive-Nationalists and Authoritarians », Party Politics, [en prépublication en ligne]: <doi:I0.1177//3540688/2472587>.

HALIKIOPOULOU D., NANOU K. et VASILOPOULOU S., 20I2, «The Paradox of Nationalism: The Common Denominator of Radical Right and Radical Left Euroscepticism », European Journal of Political Research, 5I-4, p. 504-539.

HÄUSLER A., 2013, Die "Alternative für Deutschland"- Eine rechtspopulistische Partei?, Heinrich Böll Stiftung NRW, [en ligne]: <http://www.boell-nrw.de/ sites/default/files/afd_studie_forena_hbs_nrw.pdf>.

HAWKINS K. A., 2009, «ls Chávez populist? Measuring Populist Discourse in Comparative Perspective», Comparative Political Studies, 42-8, p. I040- 1067.

HERNÁNDEZ E. et KRIESI H., 20I4, «The electoral consequences of the financial and economic crisis in Europe», working paper, European University Institute, Florence, [en ligne]:<http://www.eui.eu/Projects/POLCON/Documents/ HernandezandKriesi20|4.pdf>.

HOOGHE L., MARKS G. et WILSON C.J., 2002, «Does Left/Right Structure Party Positions on European Integration?», Comparative European Politics, 35-8, p. 965-989.

IONESCU G. et GELLNER E., 1969, Populism. Its Meanings and National Characteristics, New York, Macmillan. 
IVALDI G., 2014, «Euroscepticisme, populisme, droites radicales: état des forces et enjeux européens », L'Europe en formation, CIFE, 373, p. 7-28.

IVARSFLATEN E., 2008, «What Unites Right-Wing Populists in Western Europe? Re-examining Grievance Mobilization Models in Seven Successful Cases», Comparative Political Studies, 4I-I, p. 3-23.

JAGERS J. et WALGRAVE S., 2007, «Populism as Political Communication Style: An Empirical Study of Political Parties' Discourse in Belgium», European Journal of Political Research, 46-3, p. 319-345.

KITSCHELT H., 1995, The Radical Right in Western Europe. A Comparative Analysis, en collaboration avec A.J. McGann, Ann Arbor, University of Michigan Press.

KRIESI H. et al., 2006, « Globalization and the Transformation of the National Political Space: Six European Countries Compared», European Journal of Political Research, 45-6, p. 921-956.

-, 2008, «The Netherlands: a Challenge that Was Slow in Coming» in West European politics in the age of globalization, Cambridge, Cambridge University Press, p. 154- 182. -, 2014, «The Populist Challenge», West European Politics, 37-2, p. 36I-378.

- et PAPPAS T., 2014, «Populism in Europe During Crisis: an Introduction », [à paraître]: <http://www.eui.eu/Projects/POLCON/Documents/kriesiandpappas20|4.pdf>.

LANE Ph. R., 2012, «The European Sovereign Debt Crisis », Journal of Economic Perspectives, 26-3, p. 49-68.

LUCASSEN G. et LUBBERS M., 2012, «Who fears what? Explaining far-right-wing preference in Europe by distinguishing perceived cultural and economic ethnic threats », Comparative Political Studies, 45-5, p. 547-574.

LONG J.S. et FREESE J., 2006, Regression Models for Categorical and Limited

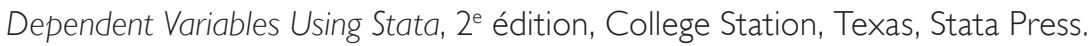
LUCARDIE P. et VOERMAN G., 2012, Populisten in de Polder, Boom, Amsterdam. MARCH L., 2007, «From Vanguard of the Proletariat to Vox Populi : Left-Populism as a "Shadow" of Contemporary Socialism », SAIS Review, 27-I, p. 63-77. -, 2011, Radical Left Parties in Europe, New York, Routledge. 
MOFFITT B. et TORMEY S., 2014, «Rethinking Populism: Politics, Mediatisation and Political Style», Political Studies, 62-2, p. 38I-397.

MOOD C., 2010, «Logistic Regression: Why We Cannot Do What We Think We Can Do and What We Can Do About It», European Sociological Review, 26-I, p.67-82.

MUDDE C., 2004, «The Populist Zeitgeist», Government and Opposition, 39-4, p.542-563. -, 2007, Populist Radical Right Parties in Europe, Cambridge, Cambridge University Press. - et ROVIRA KALTWASSER C., 2013, «Exclusionary vs Inclusionary Populism: Comparing Contemporary Europe and Latin America», Government and Opposition, 48-2, p. 147-174.

OESCH D., 2008, «Explaining Workers' Support for Right-Wing Populist Parties in Western Europe: Evidence from Austria, Belgium, France, Norway, and Switzerland», International Political Science Review, 29-3, p. 349-373.

PAUWELS T., 2010, «Explaining the Success of Neo-liberal Populist Parties: The Case of Lijst Dedecker in Belgium», Political Studies, 58-5, p. I009-1029.

ROODUIJN M., DE LANGE S. L. et VAN DER BRUG W., 2014, «A Populist Zeitgeist? Programmatic Contagion by Populist Parties in Western Europe», Party Politics, 20-4, p. 563-575.

ROVNY J., 2013, «Where Do Radical Right Parties Stand? Position Blurring in Multidimensional Competition», European Political Science Review, 5-I, p. I-26.

RYDGREN J., 2008, «Immigration Sceptics, Xenophobes or Racists? Radical RightWing Voting in Six West European Countries», European Journal of Political Research, 47-6, p. 737-765.

SAUGER Nicolas, DEHOUSSE Renaud, GOUGOU Florent, 2015, «Comparative Electoral Dynamics in the European Union in 2014 (CED-EUI4): A Data User's Guide», Les Cahiers européens de Sciences Po, n².

SPIERINGS N. et ZASLOVE A., 20I5, Special Issue, Patterns of Prejudice.

STAVRAKAKIS Y. et KATSAMBEKIS G., 20I4, «Left-Wing Populism in the European Periphery: the Case of SYRIZA», Journal of Political Ideologies, 19-2, p. I19-142.

SZCZERBIAK A. et TAGGART P., 2008, Opposing Europe? The Comparative Party Politics of Euroscepticism, Oxford, Oxford University Press. 
VASILOPOULOU S., 201I, «European Integration and the Radical Right: Three Patterns of Opposition», Government and Opposition, 46-2, p. 223-244.

VISSER M. et al., 2014, «Support for Radical left ideologies in Europe», European Journal of Political Research, 53-3, p. 541-558.

WERTS H., SCHEEPERS P. et LUBBERS M., 2013, «Euro-Scepticism and Radical Right-Wing Voting in Europe, 2002-2008: Social Cleavages, Socio-Political Attitudes and Contextual Characteristics Determining Voting for the Radical Right», European Union Politics, 14, p. 183-205.

WEYLAND K., 200I, «Clarifying a Contested Concept: Populism in the Study of Latin American Politics», Comparative Politics, p. I-22. 